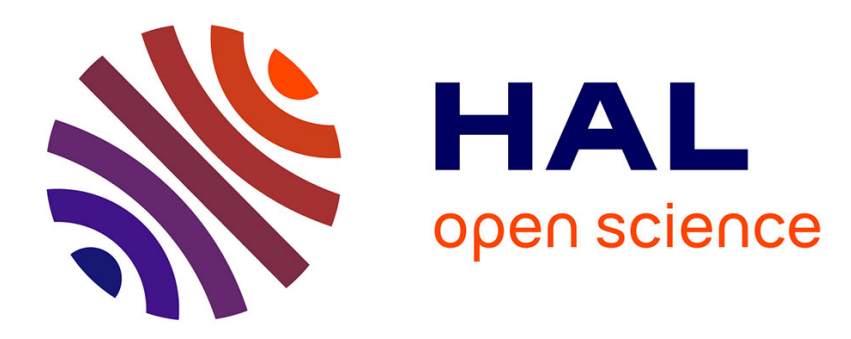

\title{
De la démocratie de négociation à la démocratie délibérative - débats théoriques et trajectoires nationales
}

Olivier Giraud

\section{To cite this version:}

Olivier Giraud. De la démocratie de négociation à la démocratie délibérative - débats théoriques et trajectoires nationales. Négociations, 2009, 12 (2), pp.215-227. halshs-00537688

\section{HAL Id: halshs-00537688 \\ https://shs.hal.science/halshs-00537688}

Submitted on 19 Nov 2010

HAL is a multi-disciplinary open access archive for the deposit and dissemination of scientific research documents, whether they are published or not. The documents may come from teaching and research institutions in France or abroad, or from public or private research centers.
L'archive ouverte pluridisciplinaire HAL, est destinée au dépôt et à la diffusion de documents scientifiques de niveau recherche, publiés ou non, émanant des établissements d'enseignement et de recherche français ou étrangers, des laboratoires publics ou privés. 
Olivier Giraud (Centre Marc Bloch, CNRS)

olivier.giraud@cmb.hu-berlin.de

De la démocratie de négociation à la démocratie délibérative - débats théoriques et trajectoires nationales ${ }^{1}$

résumé

Remise en cause de l'autorité de l'Etat et des modes hiérarchiques d'exercice du pouvoir, déclin des partis et organisations syndicales, crise des identités collectives et des modalités d'intégration, déficit démocratique des régulations supranationales... Le diagnostic de crise des formes traditionnelles de la démocratie se déploie dans différentes dimensions. Le concept de démocratie de négociation, qui bénéficie d'un écho important dans le monde germanique ou anglo-saxon constitue une perspective de réponse à ces crises, bien connue ailleurs dans le monde francophone, et cependant peu en France. Cet article présente brièvement les fondements historiques et théoriques de cette notion. II analyse ensuite les limites des modèles politiques de la démocratie de négociation, dans le contexte des transformations profondes des structures sociales qui se dessinent aujourd'hui. Enfin, il se réfère à l'exemple de la démocratie directe suisse pour conclure sur une défense des vertus d'un dialogue sociétal qui transite par le débat public et représente une forme viable de démocratie délibérative.

Introduction:

Les crises contemporaines de la démocratie se manifestent par des symptômes variés. Certains ont trait à des dimensions strictement politiques (montée des violences collectives, cristallisation de l'abstention dans certains groupes sociaux, remise en question plus fréquente des institutions de pouvoir d'Etat, etc.) ou à des dimensions plus socio-économiques (augmentation des désaffiliations et crises de l'intégration sociale, augmentation durable des inégalités, etc.). Conformément au diagnostic posé par Fritz Scharpf (Scharpf 2000), elles relèvent à la fois de l'expression démocratique et renvoient alors avant tout à une crise de la médiation sociale (input democracy) mais elles relèvent également des réponses et solutions que le système politique fournit à la société (output democracy). Les politiques publiques en ce qu'elles sont l'objet d'un grand nombre des médiations sociales et en ce qu'elles fournissent l'essentiel des réponses du système politique aux sollicitations sont ancrées au cœur du processus démocratique (Giraud, Warin 2008).

Au risque de les caricaturer, on pourrait résumer les débats sur les crises de la démocratie autour de l'incapacité croissante des systèmes démocratiques à remplir leur rôle d'intégration des individus et des groupes. Suivant alors l'un des enseignements fondateurs de la sociologie depuis Emile Durkheim, on pourrait dire que les sociétés, poursuivant leur mouvement de différenciation, éprouvent de plus en plus de difficultés à organiser la vie en commun de groupes bien différenciés, ce qui est l'essence même du politique. La sociologie systémique de Habermas (1973) distingue dans cette perspective l'intégration des individus au système de l'intégration du système, c'est-à-dire des composantes mêmes du système. L'intégration au système se fait par le truchement de sous-systèmes sociaux qui ont tendance à se différencier pour mieux remplir leur tâche, menaçant l'unité du tout

\footnotetext{
${ }^{1}$ Je remercie chaleureusement Yannis Papadopoulos, Fritz Sager, Yves Sintomer et Pierre-Eric Tixier pour leurs remarques sur une première version de ce texte.
} 
(Jobert, Muller, 1987). Niklas Luhmann (1998) en insistant sur l'auto-référentialité et l'enfermement croissant des systèmes sociaux montre ainsi comment la dimension de l'intégration du système est menacée. Incapables d'envisager les effets de leurs actes pour les autres groupes ou secteurs sociaux, ou autres sous-systèmes, les acteurs et organisations impliquées dans des logiques fermées seraient par définition, de plus en plus incapables de rentrer en négociation les uns avec les autres.

Pour autant, les travaux sociologiques les plus récents, notamment en France, insistent plutôt sur la crise des médiations collectives et, ainsi, sur la dimension de l'intégration des individus au système, en montrant la crise des identités (Dubar, 2000), des cultures de groupe (Lahire, 2004), ou des affiliations qui résultent de l'affaiblissement des formes classiques de socialisation. François Dubet évoque en la matière un processus de désinstitutionnalisation (Dubet, 2002). Les individus deviennent multiples ; ils sont tous des composés singuliers de cultures et d'identités, forment des assemblages peu cohérents, peu stables et peu saisissables. Du point de vue politique, ces individus ne se laissent que rarement réduire à former les bataillons de partis, de syndicats ou d'associations avec lesquelles il est cependant plus aisé de négocier (Giraud, 2005).

L'intégration du système par ses composantes organisées tout comme l'intégration des membres du système à des entités collectives forment deux mécanismes majeurs du fonctionnement de la démocratie. Dans les deux cas, les liens entre négociation et politique sont au centre de la réflexion et un grand nombre de modalités permettent l'organisation des relations entre médiation sociale et production des solutions politiques dans le cadre du fonctionnement démocratique. Un courant important de l'analyse comparée des systèmes démocratiques, abondamment discuté dans le monde anglo-saxon, mais aussi en Allemagne, aux Pays-Bas, en Suisse et en Belgique, est précisément centré sur les rapports entre démocratie et négociation. Le label «démocratie de négociation » renvoie ainsi à toute une série de formes institutionnalisées d'inscription de la négociation dans le système démocratique. Développé à la fin des années 60 comme une catégorie analytique sur la base de travaux comparatifs de régimes démocratiques existants (le Liban, les Pays-Bas, la Suisse pour l'essentiel), le modèle de la démocratie de négociation se saisit directement des tensions entre intégration du et au système et représente sans doute une source d'inspirations face aux crises contemporaines de la démocratie. Les modèles corporatistes, consenciationnalistes, de consensus se rangent entre autres derrière le terme générique de démocratie de négociation.

La première partie de cet article est une présentation et discussion de cette notion de démocratie de négociation. La trajectoire de cette notion, développée dans le champ de l'analyse comparative des régimes politiques, est alors rapidement retracée. Dans une deuxième partie, cette notion est ensuite discutée de façon critique, mais elle est aussi mise à l'épreuve de la réalité empirique. En effet, les régimes nationaux "champions » de la démocratie de négociation, voire simplement les arrangements sectoriels ou de moindre ampleur qui en relèvent sont confrontés depuis des années, eux aussi, à des crises profondes. Cette médiation démocratique organisée, formalisée se trouve ainsi elle aussi rattrapée par des dysfonctionnements majeurs. Seul un pays, considéré dans la littérature comparative traditionnelle, comme l'archétype de la démocratie de négociation, résiste largement aux échecs et crises politiques qui frappent le Liban, les Pays-Bas et la Belgique : il s'agit de la Suisse. Un regard rapide sur la République alpine nous invite alors à reconsidérer la crise de la démocratie de négociation dans le contexte des transformations des conditions d'exercice de la médiation sociale. Si les médiations sociales collectives sont en crise, en Suisse comme ailleurs, et tendent à disqualifier progressivement les règles de la démocratie de négociation, alors ce régime politique dispose d'une forme de d'organisation démocratique alternative : la démocratie directe. Cette norme politique permet de construire une évolution du régime démocratique en terme de démocratie de négociation, vers une forme délibérative, qui permet de nouvelles formes d'implications citoyennes. La troisième et dernière partie de notre réflexion sur démocratie et négociation est ainsi consacrée à l'examen des perspectives ouvertes par la démocratie délibérative. Elle s'achève enfin par une confrontation entre le modèle de la démocratie délibérative, considérée comme une évolution de la 
démocratie de négociation helvétique, et le modèle républicain français, dans lequel, la médiation sociale des intérêts est toujours plus organisée et appropriée par l'Etat.

Encadré 1 : La négociation comme forme de gouvernance

La science politique, notamment anglo-saxonne et germanophone, a beaucoup réfléchi à l'utilité du mécanisme de la négociation pour l'analyse des relations de pouvoir. La négociation peut alors être comprise comme une forme de gouvernance, c'est-à-dire une forme de coordination sociopolitique : "La coordination de l'action par la négociation repose sur des accords à caractère d'obligation qui sont le résultat d'une influence réciproque entre les parties prenantes, obtenues à l'occasion d'une communication directe » (Benz, 2007).

De ce point de vue, les différentes situations de négociation enclenchent des mécanismes sociaux spécifiques. Dans les situations de négociation au sens de bargaining, les discussions sont centrées sur l'échange, les concessions, les compensations, les décisions paquets, etc. Ces processus peuvent permettre de dégager des issues, notamment dans la mesure où chaque partenaire obtient des éléments dans les aspects qui comptent pour lui. Cela signifie que les positions initiales des parties prenantes ne sont pas véritablement susceptibles de se rapprocher. Dans des configurations centrées sur la fabrication de compromis, en revanche, le rapprochement porte bien sur les attentes et positions initiales des uns et des autres. II en va encore plus ainsi dans les situations de négociation qui relèvent du registre de l'argumentation (arguing). Dans ce dernier cas, les parties s'efforcent alors d'imposer à tous, en mobilisant des arguments normatifs en terme d'intérêt général par exemple, un cadre, une signification, une finalité à la situation de négociation et de laquelle découle, tout " naturellement », une issue qui leur sera favorable. Les approches dites cognitives en analyse des politiques publiques ont exploré les mécanismes d'imposition de référentiels ou de discours sociaux ou politiques qui renvoient en fait à une lutte pour l'hégémonie (Muller, 2000).

\section{La démocratie de négociation : un modèle théorique développé à partir de cas concrets}

La négociation est un mécanisme inscrit au cœur des enjeux politiques. La formulation, l'expression, la représentation et enfin l'articulation de la demande sociale sont ici les processus qui lient négociation et politique, ou plus précisément, négociation et démocratie. Depuis la fin des années 60 , les approches en terme de démocratie de négociation se sont développées. Ces approches font le lien entre input et output democracy, autrement dit, entre articulation de la demande sociale et politiques publiques. Elles méritent qu'on s'attache à la fois à leurs fondements théoriques et aux cas historiques à partir desquels elles ont été construites.

Jusqu'au milieu des années 60 , les modèles dominants d'analyse de la démocratie, américains pour l'essentiel, affirment que seules les sociétés homogènes au plan religieux, ethnique, linguistique ou encore qui ne sont pas divisées par des conflits idéologiques trop forts sont susceptibles d'accéder à la stabilité. Dans la seconde moitié des années 60 cependant, des politologues allemand (Lehmbruch, 1967) et hollandais (Lijphart, 1968) évoquent le cas de petits pays dont la démocratie est particulièrement stable et apaisée, et qui, cependant, présentent un niveau élevé d'hétérogénéité religieuse (Liban), linguistique (Belgique), linguistique et religieuse (Suisse), ou encore idéologique et religieuse (Pays-Bas). Des analyses minutieuses de l'histoire, des institutions, des cultures politiques de ces pays ont montré qu'il est possible de favoriser par des arrangements institutionnels mais aussi par la diffusion d'une "culture du compromis ", la résolution de clivages majeurs qui, dans d'autres contextes, occasionnent crises, violences et guerres. Ces politologues remarquent qu'il est possible de résoudre les conflits par la négociation (Czada, 2000). La démocratie proportionnelle (Lehmbruch, 1967) ou le consociationalisme (Lijphart, 1968) - ou démocratie de consensus - sont alors reconnus comme des formes d'organisation de la vie démocratique qui valorisent la modération politique, le respect des minorités ainsi que le pouvoir et la capacité des élites à négocier et imposer des compromis dans les sociétés clivées. Une série d'autres modèles non-majoritaires - régimes corporatistes ou 
régimes accumulant les points de veto - ont entre temps été identifiés comme autant de formes alternatives de « démocraties de négociation » (Czada, 2000 ; Armingeon, 2002).

Les approches en terme de " démocraties de négociation » regroupent l'ensemble des formes démocratiques institutionnalisant le partage du pouvoir entre groupes sociaux. Placés dans des situations d'interdépendance, communautés, partis, institutions, etc. sont forcés à la négociation pour maintenir leur place dans le système. Ces différents arrangements modèrent le principe majoritaire, au fondement du modèle libéral de la démocratie parlementaire, incarné par le modèle anglais de Westminster. Dans ce modèle archétypique britannique, les pouvoirs sont concentrés au sein d'un exécutif fort, aux mains d'un seul parti politique, dans le cadre d'institutions politiques centralisées qui n'entretiennent a priori aucune forme de relation privilégiée avec quelque groupe d'intérêt que ce soit. Dans les régimes de négociation au contraire, le compromis fait l'objet d'une véritable institutionnalisation entre différentes communautés d'action collective. Le corporatisme organise un partage du pouvoir entre Etat et groupes organisés stables et représentatifs (Lijphart/Crepaz, 1991). Les régimes de "checks and balances " - ou régimes à " acteurs-veto " - modèrent le pouvoir gouvernemental en attribuant des compétences à des institutions et des intérêts " indépendants » du pouvoir politique sur certains domaines de politiques publiques. Le fédéralisme - ou d'autres formes de décentralisation - fait de même entre le centre politique et des «périphéries » autonomes. Les régimes de consensus organisent ce partage du pouvoir entre des forces politiques qui représentent des segments contrastés dans la société, comme des groupes religieux ou linguistiques ou surtout aujourd'hui, des partis politiques (Lijphart, 1999).

Dans ces différents modèles, on ne recherche pas seulement l'assentiment de la majorité, mais bien l'assentiment du plus grand nombre (Ibid). Sont alors directement associés à l'exercice du pouvoir, les communautés ou les groupes sociaux dont l'insertion est compliquée par le risque de minorisation permanente dans le cas de clivages linguistiques ou religieux, ou dont les mobilisations s'organisent autour de conflits sociaux majeurs, comme ceux liés au développement du capitalisme, dans le cas du corporatisme.

Les outils institutionnels essentiels de la démocratie de négociation sont les gouvernements de larges coalitions politiques, l'autonomie des groupes les uns par rapport aux autres, la proportionnalité des modes de désignation des mandats ou des postes et la présence d'un veto mutuel. La dimension culturelle, sous la forme d'une réelle "volonté de coopérer», est une condition sine qua none au succès de ces régimes. Pour autant, le facteur culturel n'explique pas à lui seul l'émergence des démocraties de consensus. Les régimes politiques concernés - le régime des « piliers » aux Pays-Bas jusqu'aux années 80 , le régime politique suisse ou encore le régime de grande coalition, fortement corporatisé de l'Autriche - sont le résultat de fortes tensions ou d'affrontements intervenus entre des groupes de force à peu près égale et dont aucun ne peut durablement se trouver exclu du jeu ou de la participation démocratique (Lehmbruch, 1991). Le choix de la modération et du partage du pouvoir est ainsi plus un choix de Realpolitik qu'un choix généreux. La culture du compromis est une culture pragmatique et volontariste. Les raisons qui expliquent sa mise en œuvre peuvent ne pas se maintenir. Ces différents types de démocraties de négociation font par ailleurs l'objet de critiques également convergentes.

\section{Limites théoriques et crises politiques des démocraties de négociation}

En premier lieu, ces régimes introduisent des ambiguïtés dans la représentation démocratique. En poussant à des grandes coalitions stables, les régimes de consensus amoindrissent la transparence et la capacité de choix des électeurs. La grande coalition dessert de plus l'imputabilité (accountability) des choix et des responsabilités politiques. Plus encore, dans leur essence, toutes les formes de démocratie de négociation reposent sur la capacité des élites représentant des groupes sociaux à négocier entre elles les arrangements productifs et comptent ainsi sur la passivité des membres de ces groupes. Ces régimes sont ainsi souvent paternalistes (Kriesi, 1994, p.314). Enfin, par leur 
institutionnalisation de certains clivages sociaux, les démocraties de consensus délimitent la représentation des intérêts sociaux pertinents de manière beaucoup plus ferme que ne le feraient des régimes majoritaires plus fréquemment pluralistes. Les "nouveaux » groupes sociaux minoritaires minorités ethniques, minorités sexuelles, minorités politiques émergentes, etc. - ne peuvent que très difficilement prétendre pénétrer l'espace politique légitime.

En second lieu, sans rentrer dans le détail des différents cas nationaux évoqués, on ne peut éviter de remarquer que la plupart des régimes politiques de partage du pouvoir ont disparu. C'est le cas par exemple des régimes consociationnels, à l'exception de la Suisse. L'immuable grande coalition entre chrétiens- et socio-démocrates est sans doute durablement dépassée en Autriche, tout comme le calme rythme des alternances modérées entre larges coalitions tournantes dans le contexte néerlandais (Lehmbruch, 1991). Aux Pays-Bas précisément, le délitement des piliers qui organisaient la vie des individus, "du berceau au cimetière », et à travers la quasi-totalité de leurs activités (enseignement, syndicalisme, médias, prestations sociales, loisirs, etc.) a débouché sur une crise politique, sociale et identitaire profonde. Par ailleurs, les principaux accords corporatistes développés depuis les années 30 en Suède ou les pratiques d'action concertée, plus circonscrites, dans le cas allemand sont également en situation de crise depuis des années. Seul le fédéralisme demeure aujourd'hui une forme de démocratie de négociation vivante. La Belgique, autrefois régime de consensus, s'est d'ailleurs progressivement tournée vers cette modalité alternative de partage du pouvoir. En répartissant le pouvoir entre communautés, plutôt qu'en poussant les communautés à décider ensemble, la norme de coopération est, dans le cas belge, remplacée par une norme de division qui semble en contradiction avec la précédente. Dans ce cas en effet, la superposition des normes de partage de pouvoir conduit à une logique de décentralisation, de répartition et de séparation semblables aux dynamiques prévalant au Canada ou en Espagne. Au Liban, le régime de consensus a dégénéré en une répartition claniste et clientélaire des ressources de pouvoir. En Inde, la mise en place de régimes de partage du pouvoir a contribué à exacerber les tensions interethniques ou interreligieuses (Wilkinson, 2000). Contrairement à la théorie développée par Arend Lijphart (1999), les pratiques de délégation de pouvoir à des groupes constitués sur une base identitaire, n'engendrent pas forcément une culture du compromis, de la concorde et de la coopération, mais peuvent au contraire figer les identités, mobiliser les groupes et radicaliser les positions des uns et des autres.

Comment expliquer une telle déstabilisation des régimes politiques relevant de la démocratie de négociation ? Une des explications les plus plausibles porte sur la moindre capacité à mobiliser des organisations issues des clivages fondateurs des différentes formes de démocratie de négociation. Les identifications et appartenances religieuses comptaient parmi les clivages fondateurs de ces démocraties qui ont perdu de leurs capacités mobilisatrices en Occident ces dernières années. Le tournant réflexif et la montée de l'individualisme dans les sociétés occidentales s'accompagnent d'une novelle avancée de la sécularisation des sociétés et d'une disqualification croissante de l'influence du religieux sur la vie politique. De la même façon, les identifications et appartenances aux camps syndicaux et patronaux sont affaiblies jusque dans les pays phares du corporatisme. En Suède, les discordes croissantes entre syndicats du privé et du public, en Allemagne, les pertes d'adhésions et de capacités de mobilisation aussi bien des syndicats que des différents types d'associations patronales expliquent pour une large part l'échec des formes traditionnelles et vertueuses d'échange politique et de coordination économique et sociale dans ces pays (Thelen, 1994). Lorsque les capacités des clivages sociaux à prévenir le conflit ne sont plus effectives, les rigidités induites par les arrangements et les dispositifs institutionnels des démocraties de négociation, mais aussi leurs rapports ambivalents à la démocratie, peuvent les emporter.

Au-delà de la démocratie de négociation : les promesses de la démocratie délibérative ? 
Ces échecs et impasses condamnent-ils pour autant toute forme de démocratie de négociation ? Les modèles développés au $20^{\text {ème }}$ siècle n'ont-ils pas plutôt besoin d'être rénovés en profondeur? II est sans doute utile de poser la question du succès et de la stabilité de la démocratie helvétique, d'ailleurs toujours érigée en référence archétypique de la démocratie de consensus (Lijphart, 1984, 1999).

La littérature scientifique (Kriesi, 1998) avance souvent l'argument selon lequel, la non superposition des clivages - les frontières entre régions catholiques et protestantes ne correspondent pas aux frontières linguistiques - empêche une cristallisation des oppositions dans le cas helvétique. La Suisse serait ainsi génétiquement immunisée contre une dérive à la belge des institutions de consensus. Dans un article récent Adrian Vatter (2008) défend la position selon laquelle la Suisse serait devenue récemment un exemple de démocratie de consensus "moins extrême " pour devenir une démocratie de négociation traditionnelle. Cette évolution s'est opérée à ses yeux suite à la polarisation de la vie partisane, sous l'influence du populiste Christoph Blocher qui a remis en cause l'impératif de consensus, suite à la moindre fragmentation partisane dans le pays et la re-décentralisation du système politique dans son ensemble.

Deux arguments semblent ici décisifs. En premier lieu, comme l'affirme Vatter, la revitatisation du fédéralisme suisse renforce l'autonomie et le dynamisme politique potentiel des arènes cantonales au-delà des consensus fédéraux longs à construire et qui débouchent le plus souvent sur des politiques pragmatiques, techniques, et somme toute assez compassée. Les cantons sont dotés de ressources de pouvoir légales, administratives, mais aussi financières très importantes et d'une vie politique autonome, relayée par des médias locaux puissants. Des analyses entreprises dans le domaine des politiques de l'emploi (Perret et al., 2007) ou des politiques du handicap (Giraud et Lucas, 2007) montrent, du plus « libéral » au plus « social », la grande diversité des cultures et pratiques politiques cantonales.

En second lieu, si Vatter mentionne à juste titre la polarisation de la vie politique, il n'évoque pas les conséquences de cette polarisation sur le fonctionnement de la démocratie suisse, saisie comme un régime politique. Au-delà du fédéralisme en effet, la centralité de la démocratie directe et des institutions de la démocratie de négociation fait du régime politique suisse le modèle de démocratie délibérative nationale sans doute le plus abouti du monde.

La démocratie directe joue pour commencer un rôle essentiel dans la transformation des équilibres politiques et institutionnels en Suisse. En effet, si le partage du pouvoir entre différents partis et orientations politiques, caractéristique des démocraties de négociation, a pour conséquence de brouiller le message démocratique en noyant les responsabilités dans la coalition, la démocratie directe ouvre un espace politique autonome susceptible d'en corriger les défauts. Dans les arènes politiques fédérales, comme le plus souvent également cantonales et communales, les citoyens peuvent annuler, par référendum ${ }^{2}$ toute disposition légale ou au contraire, proposer une initiative constitutionnelle ${ }^{3}$. Ces procédures ont toutefois plus de chances d'aboutir lorsqu'elles sont portées par des groupes d'intérêts organisés. Depuis plusieurs décennies ainsi, des procédures de consultation pré-parlementaires intensives et extensives ont pour vocation de prévenir ces oppositions qui, sans cela, risquent de bloquer la vie politique du pays (Sciarini, 2006). Plus qu'à de la concertation sociale, ces procédures poussent à de véritables négociations sociales de grande envergure qui doivent impérativement aboutir au risque de voir les dispositions annulées. Le dialogue social enclenché a de plus pour caractéristique décisive d'être public. La nature, par définition, populaire de la démocratie directe conduit à une importante publicisation des débats. En situation référendaire, l'électorat a toujours «le dernier mot», sa mobilisation n'est jamais acquise. Elle doit se construire, par les discours, par la persuasion, et, il est

\footnotetext{
${ }^{2}$ Ces référendums sont dans certains cantons obligatoires lorsque les autorités cantonales décident d'augmenter les dépenses publiques. II suffit dans la règle de réunir un nombre réaliste de signatures (50 000 dans le cas le plus courant des référendums facultatifs fédéraux).

${ }^{3}$ Les initiatives impliquent la mobilisation de 100000 signataires. Elles peuvent être contrecarrées par une contreproposition du gouvernement fédéral.
} 
vrai, aussi par le truchement de la communication. Le débat public joue de ce point de vue un rôle d'importance croissante en Suisse.

On peut également faire l'hypothèse que la démocratie directe, dont la menace pèse continuellement sur les élites politiques explique en grande partie le renforcement du rôle du parlement suisse. Les deux chambres helvétiques - l'une représentant le peuple, désignée à la proportionnelle intégrale et l'autre désignée dans le contexte cantonal - prennent en charge, suite à la phase préparlementaire évoquée ci-avant, des négociations inter-partisanes approfondies qui ont également pour vocation d'écarter au maximum les oppositions et de participer au processus de mobilisation du consentement, le plus large possible. Le parlement suisse se trouve ainsi dans une position centrale qui lui permet d'élaborer plus efficacement le consensus, sur la base d'une négociation entre positions devenues plus tranchées, notamment dans les domaines sensibles tels ceux des politiques sociales (Papadopoulos 2008, pp. 296-297). Les recherches conduites récemment sous la direction de Yannis Papadopoulos (Ibid.) indiquent même que les négociations inter-partisanes conduites au cours de la phase parlementaire de la décision politique, tendent à supplanter en importance la phase préparlementaire centrée sur les organisations d'intérêts. La forte capacité des partis politique à agréger les intérêts et positions en provenance à la fois de la sphère politico-institutionnelle, mais aussi des intérêts, ou encore, dans certains champs de pouvoir, d'être en prise avec des instances internationales dont le rôle s'est renforcé depuis les années 90, expliquerait alors ce renforcement des partis. Cependant, cette centralité retrouvée des partis ne s'explique pas vraiment en dehors de l'impératif de construction d'un consensus lié à la démocratie directe : l'ombre de la démocratie directe permet à mon sens d'expliquer, la dimension délibérative du fonctionnement du processus démocratique parlementaire helvétique. La délibération dans le cas suisse prend la forme d'une négociation politique et sociale, fortement publicisée, dans le but non seulement de réduire les oppositions, mais aussi de mobiliser le consentement.

Les transformations des grandes structures sociales que sont la désaffiliation des individus à des ensembles sociaux et la difficulté croissante à intégrer les différents sous-ensembles du système sont des dynamiques sociales qui ne semblent pas avoir d'impact négatif sur la conduite du débat public. Au contraire, le peuple et ses réactions électorales deviennent pour une part sans doute moins prévisibles, mais la désaffiliation ne débouche pas sur une hausse équivalente de l'indifférence politique ou de l'abstention. La participation populaire aux votations fédérales reste ce qu'elle a été depuis le milieu des années 1950, c'est-à-dire faible et située en moyenne entre 40 et $50 \%{ }^{4}$. En revanche, la polarisation du débat public a sans doute beaucoup contribué à ouvrir les motifs du choix électoral des citoyens. La rupture notamment entre une droite populiste xénophobe, incarnée par l'ancien Conseiller Fédéral Blocher et les socio- ou chrétiens-démocrates a rendu impossible le consensus minimal et encore plus les alliances et coalitions politiques : le récent évincement de Blocher du conseil fédéral l'illustre bien. L'affaiblissement des capacités du bloc partisan représenté au gouvernement fédéral, selon la mythique formule magique ${ }^{5}$ à produire une position officielle a ouvert un espace à une offre politique polarisée et à des comportements politiques dans l'arène référendaire qui le sont tout autant.

La démocratie directe helvétique renouvelée confère ainsi une dimension délibérative conséquente aux actes démocratiques qui se déroulent dans son aire d'exercice. La Suisse emprunte ainsi un chemin qui la mène d'une démocratie de consensus vers une démocratie délibérative populaire. Les vertus d'un débat public sanctionné par le vote - et non confisqué par des professionnels de la captation de la démocratie délibérative ou participative comme c'est souvent le cas en France (Blondiaux, Sintomer 2002) - sont prometteurs en Suisse, que cela soit au sein de l'arène fédérale ou au sein des arènes cantonales.

\footnotetext{
${ }^{4}$ http://www.bfs.admin.ch/bfs/portal/fr/index/themen/00/02/sektoriel/03 06/03 $06 \quad 01 . h t m l$

${ }^{5}$ La coalition a ratissé très large entre 2003 et 2007 puisque socio-démocrates, chrétiens-démocrates, libéraux et extrêmedroite ont cohabité au sein d'un même gouvernement.
} 
Alors qu'en Suisse, la démocratie directe contribue au bon fonctionnement des institutions de démocratie de négociation ouvrant la perspective d'une démocratie délibérative populaire, la situation dans un pays marqué par le régime républicain majoritaire, comme la France, se caractérise par des tendances contradictoires. Depuis le milieu des années 90 , des spécialistes français de l'analyse des politiques publiques (Jobert, 1995) déplorent la disparition progressive de la concertation sociale en France et l'appropriation de l'expertise et du débat public, notamment dans le domaine clé des politiques sociales et de l'emploi, par des clans d'experts d'Etat, tous plus ou moins formés selon le même moule, celui de la statistique publique et de l'analyse économétrique. Cette situation n'a fait qu'empirer depuis (Barbier, 2008 ; Gauron, 2008). Dans le même temps, les consultations syndicales et patronales ne sont plus qu'une figure rituelle, le conseil économique et social ne pèse plus rien et le Plan qui était un lieu central de concertation sociale nationale a été supprimé... L'exclusion systématique des intérêts constitués de l'arène politique n'est par ailleurs pas inscrite en France dans un projet démocratique alternatif. Elle est plutôt le signe d'une exacerbation du pouvoir d'un Etat central qui se différencie et s'isole au sein de la société. La mobilisation des intérêts sociaux, leur mise en présence, leur expression sont cependant la condition du maintien de formes de négociation dans l'espace démocratique, surtout quand il reconnaît comme c'est le cas en France, aussi peu de place à des institutions qui organisent le partage du pouvoir. Même si les organisations collectives s'affaiblissent, la représentation des intérêts sociaux permet à une forme de "vérité » des attentes sociales des uns et des autres de se faire jour. C'est tout particulièrement le cas dans l'univers des politiques de l'emploi et de la protection sociale où les " experts " parlent aujourd'hui successivement au nom des familles, des pauvres, des entreprises ou des collectivités locales. A ne reconnaître aucun espace de pouvoir aux forces sociales et à se contenter de dessiner des anticipations sur la base de modèle économétriques, les administrations centrales courent le risque d'accumuler fausses interprétations et dispositifs mal conçus (Gauron, 2008).

En revanche, le dialogue social s'est en France territorialisé (Jobert 2003). II rejoint ainsi les espaces d'expression démocratique plus ouverts nés de la décentralisation. Les politiques y sont cependant plus souvent mises en œuvre que véritablement décidées. II y est donc plutôt question d'output que d'input democracy. Les réseaux, les diverses formes de l'échange politique territorialisé (Négier, 2008) ou ces pratiques de dialogue social territorial sont cependant autant d'indications du fait que la France pratique la démocratie de négociation de façon croissante, mais précisément dans un espace où input et output democracy sont déconnectées (Papadopoulos 2008). II faut alors compter sur la capacité d'entraînement des organisations et procédures collectives pour limiter les déficits de légitimité qui pourraient ressortir de ce découplage... ce qui n'est jamais si évident dans le cas français. Comme en de nombreux registres au demeurant, il semble qu'en France, les «territoires », les espaces démocratiques locaux et régionaux, doivent tenter de compenser les crispations occasionnées par la raideur et, souvent, l'inadaptation des politiques nationales, causées par les désajustements croissants du fonctionnement des institutions politiques vis-à-vis des attentes démocratiques.

Que retirer sur un plan plus réflexif de cette ébauche de comparaison entre la Suisse et la France, du point de vue des couplages entre régime démocratiques et régime de politiques publiques? II semble, mais la réflexion est ici à peine esquissée, qu'il serait utile de distinguer différentes formes démocratiques en fonction à la fois des modalités de participation des individus dans le processus démocratique et de la distance des individus à l'exercice du pouvoir effectif de décider. En premier lieu, la démocratie élective est largement ouverte pour ce qui concerne les modalités de participation. Tous les citoyens participent certes, mais ils restent très éloignés de l'exercice du pouvoir de décision sur des enjeux concrets. II s'agit de l'archétype du modèle de la représentation. En second lieu, la démocratie de négociation permet la participation des individus via leur adhésion à des organisations collectives 
comme des syndicats ou des communautés constituées. En tant que tels, ils disposent d'un pouvoir, spécifique, comme membres de leur organisation collective. Ce pouvoir de membres leur permet d'agir sur les stratégies de l'organisation vis-à-vis de l'extérieur, et peut porter sur des enjeux concrets. Les conditions de la participation sont ainsi plus restreintes, mais la capacité à agir sur les décisions est potentiellement plus forte que dans le modèle précédent. Enfin, en troisième lieu, la voie de la démocratie directe est celle à travers laquelle la participation équivaut à l'exercice, certes dilué et circonscrit, d'un véritable pouvoir de décision.

Associant extension des droits à participer et extension du pouvoir de décider, la démocratie directe présente surtout l'avantage de déclencher un processus délibératif qui concerne tous les citoyens dans la société. Les défauts, obstacles, contradictions des procédures délibératives ne doivent pas être ignorés (Held, 2006, p. 241). Le cas suisse, où pendant longtemps la démocratie directe a surtout servi les intérêts de conservateurs mieux organisés, mobilisés, et dotés de moyens de communication important, rappelle d'ailleurs en partie la réalité de ces limites. Cependant, la polarisation du débat public vient dans ce pays compenser heureusement l'affaiblissement des moteurs de la démocratie de négociation que sont les groupes d'intérêts et les partis, et qui est due, là comme ailleurs sans doute à la crise des identités collectives et à la désaffiliation. La dimension participative et populaire de la démocratie directe permet dans ce contexte d'occuper un espace essentiel. Les groupes d'intérêts et partis continuent pour leur part de jouer le rôle clé d'animateurs de la délibération et des négociations sociales, à l'ombre du verdict populaire.

$\underline{\text { Références bibliographiques: }}$

ARMINGEON, Klaus (2002), "The effects of negotiation democracy: A comparative analysis". European Journal of Political Research, 41, pp. 81-105.

BARBIER, Jean-Claude (2008). La transformation des forums de politique sociale en France. GIRAUD, Olivier et WARIN, Philippe (dirs.), Politiques publiques et démocratie. Paris, La Découverte, pp. 217240.

BENZ, Arthur (2007), "Verhandlungen" BENZ, Arthur ; Susanne LÜTZ; Uwe SCHIMANK ; Georg SIMONIS, (2007) Handbuch Governance - Theoretische Grundlage und empirische Anwendungsfelder, Wiesbaden: VS-Verlag, p. 106-119.

BLONDIAUX, Loïc ; SINTOMER, Yves (2002), "L'impératif délibératif". Politix, 15, (57), pp. 17-35.

CZADA, Roland (2000), Dimensionen der Verhandlungsdemokratie Konkordanz, Korporatismus, Politikverflechtung, polis Nr. 46.

DUBAR, Claude (2000), La crise des identités - l'interprétation d'une mutation. Paris: PUF.

DUBET, François (2002), Le déclin de l'institution. Paris: Seuil.

DURAN, Patrice (1999), Penser l'action publique. Paris: LGDJ.

GAURON, André (2008), "Un cas d'école de changement de référentiel : la politique d'exonération de cotisations sociales", dans GIRAUD, Olivier et WARIN, Philippe (dirs.), Politiques publiques et démocratie. Paris, La Découverte, pp. 199-217.

GIRAUD, Olivier (2005), « Négociation et action collective en politique - Les institutions et la culture du compromis politique face à la mutation des formes de la conflictualité sociale ». In NACHI, Mohamed et DE NANTEUIL, Matthieu (dirs.) Eloge du compromis - Pour une nouvelle pratique démocratique. Académia-Bruylant, Bruxelles, pp. 287-306. 
GIRAUD, Olivier et LUCAS, Barbara (2007) « Jeux d'échelles et référentiels dans les politiques du handicap en Suisse ». FAURE, Alain ; LERESCHE, Jean-Philippe ; MULLER, Pierre ; NAHRATH, Stéphane (dirs.), Action publique et changement d'échelle : les nouvelles focales du politique. Paris, L'Harmattan, pp. 119-134.

HABERMAS, Jürgen (1973), Legitimationsprobleme im Spätkapitalismus. Francfort sur le Main: Suhrkamp. [trad. française : "Raison et légitimité"].

HABERMAS, Jürgen (1992), Faktizität und Geltung. Francfort sur le Main: Suhrkamp.

JOBERT Anette (2003), Quelle régulation dans l'espace territorial ? in G. DE TERSSAC (ed), La théorie de la régulation sociale de Jean-Daniel Reynaud : débats et prolongements, Paris, La Découverte, collection Recherches, pp.135-146.

JOBERT, Bruno (1995), "De la nécessaire incohérence de l'action étatique", dans THERET, Bruno, L'Etat, la finance et le social. Souveraineté nationale et construction européenne. Paris : La Découverte, janvier 1995, p. 223-252 (collection Recherches)

JOBERT, Bruno (1998), "La régulation politique : le point de vue d'un politiste". COMMAILLE, Jacques ; JOBERT, Bruno (dir.), Les métamorphoses de la régulation politique. Paris: LGDJ, pp. 119-144.

KRIESI, Hanspeter (1994), Les démocraties occidentales - Une approche comparée. Paris: Economica. KRIESI, Hanspeter (1998). Le système politique suisse. 2e édition, Paris, Economica.

LAHIRE, Bernard (2004), La Culture des individus. Dissonances culturelles et distinction de soi, Paris, La Découverte.

LEHMBRUCH, Gerhard (1967), Proporzdemokratie: Politisches System und politische Kultur in der Schweiz und in Österreich. Tübingen: Mohr/Siebeck.

LEHMBRUCH, Gerhard (1991), "Das konkordanzdemokratische Modell in der vergleichenden Analyse politischer Systeme". MICHALISKY, Helga (dir.), Politischer Wandel in konkordanzdemokratischen Systemen. Vaduz: Verlag der der Liechtensteinischen Akademischen Verlag, pp. 13-24.

LIJPHART, Arend (1968), "Typologies of Democratic Systems". Comparative Political Studies, 1, pp. 344.

LIJPHART, Arend, 1984: Democracies. Patterns of Majoritarian ans Consensus Government in

Twenty-One Countries. New Haven: Yale Univ. Press.

LIJPHART, Arend (2002), "Negotiation democracy versus consensus democracy: Parallel conclusions and recommendations". European Journal of Political Research, 41, pp. 107-113.

LIJPHART, Arend ; CREPAZ, Markus M.L. (1991), "Corporatism and Consensus Democracy in Eighteen Countries: Conceptual and Empirical Linkages". British Journal of Political Science, 21, pp. 235-246.

LUHMANN, Niklas (1998), Die Gesellschaft der Gesellschaft, Frankfurt a.M.: Suhrkamp.

MULLER (Pierre), « L'analyse cognitive des politiques publiques, vers une sociologie politique de l'action publique », Revue française de science politique, 50 (2), 2000. uverte.

PAPADOPOULOS, Yannis (2008), dans GIRAUD, Olivier et WARIN, Philippe (dirs.), Politiques publiques et démocratie. Paris, La Découverte, pp. .

SCHARPF, Fritz (2000), Gouverner l'Europe, Paris, Presses de Sciences Po.

SCIARINI, Pascal (2006), "Le processus législatif" dans KLÖTI, Ulrich; KNOEPFEL, Peter; KRIESI, Hanspeter; LINDER, Wolf; PAPADOPOULOS, Yannis; SCIARINI, Pascal, Handbuch der Schweizer Politik, Zürich, Verlag Neue Zürcher Zeitung, p. 491-526.

THELEN, Kathleen (1994) "Beyond Corporatism: Toward a New Framework for the Study of Labor in Advanced Capitalism," Comparative Politics 27.

VATTER, Adrian (2008). Vom Extremtyp zum Normalfall? Die schweizerische Konsensusdemokratie im Wandel: Eine Re-Analyse von Lijpharts Studie für die Schweiz von 1997 bis 2007 pp. 1-47(47) RSSP volume $14, n^{\circ} 1$.

WILKINSON, Steven lan, 2000. India, Consociational Theory and Ethnic Violence. Asian Survey, 40, 5. 767-791. 
